OBETS. Revista de Ciencias Sociales

Vol. 16, no 2, 2021, pp. 449-464

ISSN: 1989-1385

https://doi.org/10.14198/OBETS2021.16.2.14

\title{
MÁS ALLÁ DEL AMOR: TRAYECTORIAS LABORALES Y RESPONSABILIDADES DOMÉSTICAS EN PAREJAS BINACIONALES HETEROSEXUALES ALTAMENTE CUALIFICADAS DE ESPAÑOLES/AS Y LATINOAMERICANOS/AS
}

\author{
BEYOND LOVE: WORK TRAJECTORIES AND DOMESTIC RESPONSIBILITIES IN BINATIONAL \\ HETEROSEXUAL COUPLES OF HIGHLY SKILLED SPANIARDS AND LATIN AMERICANS
}

\section{Jordi Roca Girona}

Universitat Rovira i Virgili, España

jordi.roca@urv.cat

https://orcid.org/0000-0002-5125-5607

\author{
Claudia María Anleu-Hernández \\ Universitat Rovira i Virgili, España \\ claudiamaria.anleu@urv.cat \\ https://orcid.org/0000-0001-8459-3701
}

\author{
Verónica Anzil \\ Universitat Rovira i Virgili, España \\ veronica.anzil@urv.cat \\ https://orcid.org/0000-0001-5968-4898
}

Cómo citar / Citation: Roca, J.; Anleu-Hernández, C. A. y Anzil, V. (2021) "Más allá del amor: Trayectorias laborales y responsabilidades domésticas en parejas binacionales heterosexuales altamente cualificadas de españoles/as y latinoamericanos/as". OBETS. Revista de Ciencias Sociales, 16(2): 449-464. https://doi.org/10.14198/OBETS2021.16.2.14

\section{(C) 2021 Jordi Roca Girona, Claudia María Anleu-Hernández, Verónica Anzil}

Este es un artículo de acceso abierto distribuido bajo los términos de la licencia de uso y distribución Creative Commons Reconocimiento 4.0 Internacional (CC BY 4.0) https://creativecommons.org/licenses/by/4.0/deed.es

Recibido: 02/06/20. Aceptado: 18/01/21

\section{Resumen}

Abordamos el tema de las migraciones de carácter amorosomatrimonial, centrándonos en mujeres y hombres migrantes altamente cualificadas/os. Analizamos la incidencia que han tenido las migraciones de Sur a Norte como viceversa en las carreras laborales y en la gestión del ámbito doméstico de parejas binacionales heterosexuales formadas por un/a español/a y un/a latinoamericano/a. Basándonos en diversos proyectos de investigación cualitativa, estudiamos el balance entre la esfera laboral y la doméstica/reproductiva. En lo profesional, constatamos una degradación de condiciones laborales. En lo doméstico, constatamos un aumento en responsabilidades del hogar y/o cuidado de los hijos, especialmente para las mujeres.

Palabras clave: Parejas binacionales; trayectorias laborales; roles domésticos; género; trabajo cualificado.

\begin{abstract}
We address the issue of love-marriage migration, focusing on highly skilled migrant men and women. We examine the impact of South to North and vice versa migration on the careers and in the household management of heterosexual binational couples formed of a Spaniard and a Latin American. Based on several qualitative research projects, we studied the balance between both the working and the domestic/ reproductive spheres. We observed a deterioration on the working conditions while in the domestic sphere, we detected increased household and/or childcare responsibilities, particularly accentuated in the lives of female interviewees.
\end{abstract}

Keywords: Binational couples; work trajectories; domestic roles; gender; highly skilled work. 


\section{Extended abstract}

Our study addresses the general field of migrations; specifically, the effects of migration on the careers and household management of heterosexual binational couples formed of a Spanish and a Latin American. The originality of our contribution lies in the study of love-marriage migration as opposed to economic migration, plus mobility in the northsouth direction in addition to the usually considered southnorth path, and the focus onto highly qualified migrants. The main objective was to examine the impact of migration on the professional trajectories and household management of binational couples, in terms of the balance between both the labour and the domestic/reproductive spheres. Although there is specific literature on each of these topics, we do not know of any study that brings them together so far, as we do in our contribution.

Most of our protagonists share the following characteristics: they are qualified people, with university degrees who, at some point of their lives met a person of another nationality who lived in another country (or who was temporarily staying in the same country as them). They decided then to start a romantic relationship that implied their migration to their partner's place of origin. Another common point is their connection to Spain, which is the country they migrated from or to. Thus, participants were subdivided into two sub-groups: one including people who migrate from Latin America to Spain - a group that has been widely studied - and the other formed by people with the opposite migratory route - which has been very little considered in recent scientific literature.

Our methodological approach has been eminently qualitative with an ethnographic base as we consider it to be the most suitable one for accessing the field of the private, interpersonal and gender relations. It is also the most appropriate approach to obtain descriptive and subjective information about work itineraries as well as management and distribution of the domestic and reproductive activities of the people involved, from an emic point of view.

The selection of participants was carried out following the snowball technique. Without seeking statistical representativeness but rather to achieve a deeper understanding of gender relations connected to the working and domestic environment among these couples, we selected 18 people from more than 150 interviewees. These interviews result of previous research projects which studied different aspects of binational couples' experiences. The main criteria for selecting the sample was the Latin American origin of one of the spouses while the other had to be Spanish. Among the general sample, we selected the accounts of those couples where the topics of work trajectories and the division of domestic labour were extensively addressed, as well as the fact that they were formed of at least one highly qualified member. The resulting sample is formed of 11 women (six Spanish, two Mexican, one Cuban, one Peruvian and one Venezuelan) and 7 men (six Spanish and one Mexican).

In order to collect the data, we conducted face to face indepth interviews - except for one carried out on Skype - in one or more sessions in Spain (mainly in Tarragona and
Barcelona), Mexico and Brazil. The fieldwork was developed within four projects of the Spanish National Research Plan between 2006 and 2018, all led by one of the authors. All interviews were digitally recorded (prior informed consent signed by all the participants and their respective partners), transcribed verbatim, and coded for later analysis.

The analysis of the information was also carried out in a qualitative way within the framework of grounded theory and analytical induction. We first coded and organized the transcribed interviews according to the place of origin of each of the spouses. We then created categories around the transformations in gender relations and the work trajectories of the interviewees and their spouses. Having compared their discourses and perceptions, we identified the most significant issues for a better understanding and analysis, considering previous theoretical contributions in those topics.

The results are partly consistent with what other authors have pointed out in studies with qualified migrant couples of the same nationality. But they also provide some new data and nuances, especially in connection with the subgroup of qualified women from the "global north" who migrated for love to the "global south".

A very relevant aspect is that, regardless of the economic situation of either their country of origin or destination, and their nationality, most interviewed women made their decisions placing their working and professional projects onto a secondary level, giving priority to their spouses'. Apparently, the influence of gender segregation based on the production-reproduction polarity is still very present. We also observed that among women in many cases love prevails over their paid work and professional careers, while the opposite is true for most men. Moreover, many men impose - in a more or less subtle way - the migration of their female partners as a condition in order to continue their sentimental relationship. Thus, men end up in an advantageous position, as they are "at home", while women are not familiar with the culture of their host country. Besides, many of our protagonists quitted a good job position for love but all and every one of them accepted a downgraded one after emigrating, negatively affecting their professional career. As a result, social and cultural capital of these qualified women migrating for love decreases, while their spouses' does not significantly differ.

Our contribution also provides an innovative finding on the yet scarcely studied process of male love migration. More specifically, of an aspect not yet addressed by the specialized literature, such is the love migration of men from the "global north" to the "global south". In some cases, this migration occurs at a later stage of the sentimental relationship, which differs to the initial and more usual wife's migration to the husband's country of residence. But in other cases, love migration of the male partner to the country of the female partner turns out to be the first option. Anyhow, in most cases migrant men are also affected by similar consequences pointed out for women: difficulty in entering the labour market, reduced income, downgraded prospects for professional trajectories, overqualifications for the available jobs, underemployment or unemployment. In addition, we noticed their increasing participation and responsibility on 
reproductive tasks - basically on house chores and childupbringing and care- but in a lesser proportion than the ones we observed among women. Concerning to this matter, we have been able to confirm that the greatest resistance does not necessarily come from men originally from the "global south". Coming from the "global north" is no guarantee of a better disposition and attitude to assume greater domestic responsibilities. Similarly, sometimes men's willingness to become more involved in the reproductive sphere is held back in part by what might be called the predominant gender system in the host society.

\section{INTRODUCCIÓN}

Las condiciones actuales de la globalización han permitido la ampliación del campo matrimonial (Illouz, 2012) y, por tanto, la posibilidad de encontrar pareja en cualquier lugar del mundo. Como la cohabitación y la co-residencia siguen siendo elementos estructurales de la pareja contemporánea (Beck y Beck-Gernsheim, 2012), la culminación lógica de estas parejas transnacionales comporta la migración de uno de los miembros al país del otro. Además de la nueva convivencia con el otro amado, mediada por variados procesos interculturales, ¿qué consecuencias se derivan de este proceso migratorio y de la conformación de una pareja binacional en las trayectorias laborales de ambos miembros cuando pertenecen a la categoría de altamente cualificados? Y ¿cómo se organiza la gestión de la esfera doméstico-reproductiva en una pareja a menudo socializada en sistemas de género diferentes?

El artículo se enmarca en el campo general de las migraciones y, más concretamente, en la incidencia de éstas en la configuración de la relación entre la carrera laboral y las responsabilidades domésticas de parejas heterosexuales binacionales con un miembro español y otro de un país latinoamericano ${ }^{1}$. Si bien estos temas han sido ampliamente tratados por separado por la literatura especializada, nuestra aportación posee un carácter novedoso por tres razones: porque focaliza la atención en parejas en las que uno de los miembros ha realizado una migración principalmente por razones amorosas; porque no sólo prestamos atención a parejas en las que el miembro migrante proviene del Sur global sino que incorporamos otras con el migrante procedente del Norte global; y porque la mayoría de los miembros de estas parejas son altamente cualificados. Así, nos centramos en un grupo que no responde

1 Hemos aplicado un concepto de pareja amplio que no discrimina entre las legalmente constituidas y las de hecho, en tanto que los datos estadísticos disponibles se refieren a uniones legales. al perfil mayoritariamente estudiado de migrante económico poco/no cualificado del Sur global.

El objetivo central del estudio es examinar la incidencia que han tenido las migraciones en las carreras laborales y en la gestión de la esfera doméstica de las parejas binacionales atendiendo a dos variables fundamentales: el género y la cualificación profesional. Si bien existe literatura más o menos específica sobre cada una de estas variables, no conocemos ningún trabajo que las aúne, tal y como lo hacemos en nuestra aportación.

Partimos de la hipótesis que el impacto en la trayectoria laboral será menor en el miembro local de la pareja y/o en el hombre, y que las tareas domésticas recaerán principalmente en la mujer, especialmente si existen hijos pequeños. Si bien la persona que migra por amor posee cierta ventaja en la incorporación a la sociedad de destino (Roca, Soronellas, Bodoque, 2012) respecto a quienes migran por razones laborales, a menudo entre ellas no se encuentra la de la incorporación laboral. Buena parte de la migración femenina amorosa admite la expectativa de sus futuras parejas masculinas de centrarse en la esfera reproductiva, en el marco de un sistema de género basado en la división del trabajo sostenida por la figura del hombre proveedor y de la mujer ama de casa (Constable, 2003; Robinson, 2007; Williams, 2010).

\subsection{Migraciones amorosas}

Los estudios clásicos sobre las migraciones desde las Ciencias Sociales se centraron de forma exclusiva en las causas de naturaleza económica y laboral de estos movimientos poblacionales (Harris y Todaro, 1970), así como en su dimensión individual y mayoritariamente masculina.

En las últimas décadas, el nuevo orden global ha facilitado la delimitación de nuevas geografías y tipologías de migración: migraciones de crisis, de mujeres independientes, de profesionales y 
trabajadores cualificados, de estudiantes, de jubilados, medioambientales, etc. (King, 2002), entre las cuales se encuentran las denominadas "migraciones por amor" (Roca, 2007).

Penny y Khoo (1996) pusieron el foco hace tiempo en el amor como factor motivacional de la migración. De hecho, la migración por motivos amorosos ha existido siempre, pero en la década de los noventa del siglo pasado este fenómeno aumenta de forma importante y se visibiliza más, dando lugar a las denominadas rutas globales de búsqueda de pareja. Estas rutas establecen conexiones fundamentalmente entre hombres de países del "Norte global" con mujeres del "Sur global".

Esta creciente dimensión del fenómeno ha dado lugar a la aparición de un buen número de trabajos que abordan diversos aspectos de las parejas binacionales. El hecho que estén vinculadas a menudo a procesos de migración no laboral/económica ha dejado estos aspectos en un segundo plano en favor de otros relacionados, por ejemplo, con los contextos legales de la migración, las motivaciones y las estrategias para la búsqueda transnacional de pareja, las relaciones familiares transnacionales, los procesos de aculturación, integración e interculturalidad de los miembros de la pareja y de los hijos de éstas, las políticas globales del amor, la economía política del deseo, las relaciones conyugales y su éxito o fracaso, los procesos de negociación y gestión de la identidad, las cuestiones relativas a la ciudadanía, etc. ${ }^{2}$

\subsection{Migraciones cualificadas}

No existe un consenso internacional sobre la categoría de "profesional cualificado", aunque los estudios sobre migración internacional cualificada se centran en las élites corporativas transnacionales, caracterizadas por su elevado índice de masculinización (Kofman, 2000). Más aún, las personas migrantes internacionales cualificadas tienden a ser vistas como si se tratara de seres "sin género" que no forman parte de un hogar y cuyas experiencias están puramente relacionadas con el empleo remunerado (Kofman, 2000; Willis y Yeoh, 2003), sin considerar la intersección familiar (Varrel, 2011). En estos estudios, las mujeres y las relaciones

2 Los textos recopilatorios de Breger y Hill (1998) y de Charsley (2012), así como los de carácter general de Constable (2003) y Williams (2010) proporcionan una buena muestra de esta diversidad temática. de género padecen una patente invisibilidad y/o una general representación como "esposas dependientes", al margen de su cualificación (Purkayastha, 2005; Riaño y Baghdadi, 2007). La poca literatura existente (Aure, 2013) se fija en las profesionales demandadas por los países del "Norte global".

En los trabajos más específicamente centrados en la trayectoria laboral de mujeres migrantes cualificadas predomina la presencia de la movilidad de Sur a Norte y la ausencia de la dimensión de género (Beiruti, 2008; Iredale, 2000). En ellos se pone de relieve el impacto negativo sobre sus carreras profesionales mediante la activación de procesos de descualificación y reorientación doméstica, con reducción de su actividad laboral a jornada de tiempo parcial e intensificación del trabajo doméstico (Ho, 2006; Meares, 2010), o incluso subocupación, subempleo y desempleo (Cooke, 2007; Man, 2004). La mayoría de los trabajos son cuantitativos y centrados en países que seleccionan inmigrantes usando la cualificación como uno de los criterios, tales como Australia (Ho y Alcorso, 2004), Canadá (Man, 2004) y algunos países de Europa (Dumont, Martin, y Spielvogel, 2007). Algunas de estas investigaciones se centran en la migración cualificada de parejas que forman hogares de doble carrera y muestran cómo, cuando el proceso migratorio es liderado por el marido cualificado, el aumento de la carga doméstica en la esposa cualificada es muy notorio, mientras que en la situación inversa el trabajo doméstico lo realiza personal contratado (Yeoh y Willis, 2005; Willis y Yeoh, 2003;). Hardill y MacDonald (1998) inciden sobre la búsqueda de la "recolocación" en el nuevo lugar de destino, al que dedicaremos también nuestra atención.

Las aportaciones sobre migración de índole amorosa de personas altamente cualificadas son escasas. Podemos señalar los trabajos de Cam Thai $(2002,2005)$, titulado muy significativamente "Clashing Dreams", sobre esposas vietnamitas con un alto nivel educativo y maridos estadounidenses de baja cualificación laboral, y los de Yeoh y Willis (2005), y Willis y Yeoh (2003) que interrelacionan género, matrimonio y migración cualificada de personas de Singapur a China.

Contamos, pues, con trabajos que abordan algunos de los temas de nuestra aportación, pero no de una manera conjunta. En este artículo nuestra ambición es articular la migración amorosa -tanto femenina como masculina y en sentido Sur-Norte como Norte- 
Sur- de personas con alta cualificación para evaluar la incidencia que tal movilidad tiene sobre sus carreras laborales y sobre su implicación en los procesos de reproducción.

\section{METODOLOGÍA}

Nuestro abordaje metodológico ha sido de carácter eminentemente cualitativo de base etnográfica, por considerarlo el más adecuado para adentrarse en el campo de lo privado, de las relaciones interpersonales y de género.

La selección de informantes se hizo siguiendo la técnica de la bola de nieve. Realizamos entrevistas guiadas en profundidad presenciales -a excepción de una hecha vía Skype- en una o dos sesiones, en España (principalmente en Tarragona y Barcelona), México y Brasil, en el marco de lo que podríamos llamar una etnografía multisituada (Marcus, 1995). Las personas entrevistadas eligieron uno de los formatos de entrevista propuestos: individuales (a un solo miembro de la pareja); conjuntas (a ambos miembros de la pareja simultáneamente); y dobles (entrevista individual a ambos). Todas las entrevistas fueron grabadas (previo consentimiento informado), transcritas de manera literal, y codificadas para su posterior análisis.

El trabajo de campo se desarrolló en el seno de diversos proyectos de investigación ${ }^{3}$ centrados en

3 Las referencias de los proyectos del Plan Nacional de I+D+I de España son: 1. 'Amor importado, migrantes por amor: la constitución de parejas entre españoles y mujeres de América latina y de Europa del Este en el marco de la transformación actual del sistema de género en España' (47/05): 2006-2008 'AMORIMPORT'; 2. 'Amores transnacionales: constitución y desarrollo de parejas mixtas en España' (CSO2009-10187): 2010-2012 - 'AMORMIXT'; 3. 'Parejas mixtas residentes fuera de parejas binacionales heterosexuales con uno de los miembros de nacionalidad española. Las principales temáticas abordadas en estos proyectos fueron: expectativas y motivaciones de elección de una pareja extranjera, modos de búsqueda de dicha pareja, la fase de noviazgo, la decisión de formalización de la pareja y de migración de uno de los miembros al país del otro, relación de pareja y de ésta con las respectivas familias de orientación, trayectorias laborales de ambos miembros de la pareja, la decisión sobre tener hijos y su socialización y, en su caso, fin de la relación y sus consecuencias.

Sin intención de buscar representatividad en términos estadísticos, pero sí de conseguir una comprensión más profunda de las relaciones de género vinculadas al ámbito laboral en las parejas binacionales estudiadas, seleccionamos un total de 18 personas del conjunto de las más de 150 personas entrevistadas en el marco de los proyectos anteriormente mencionados.

El criterio principal para la selección de nuestra muestra era que uno de los miembros fuese de nacionalidad latinoamericana y otro española, en tanto que se trata de la conformación más frecuente entre las parejas binacionales con un miembro español. De entre la muestra de este tipo de parejas retuvimos aquellas en las que el relato sobre las trayectorias laborales y la división del trabajo doméstico era extenso y, obviamente, que estuvieran formadas por al menos una persona altamente cualificada (Cuadro 1).

España: relaciones de género, dinámicas sociales y conexiones transnacionales' (CSO2012-33565): 2013-2015 - 'EXTRAMIXT'. 4. 'Roles de género e interculturalidad de las parejas mixtas en España: una investigación cualitativa longitudinal' (CSO201565531-P): 2016-2018 - 'LONGMIXT'. 

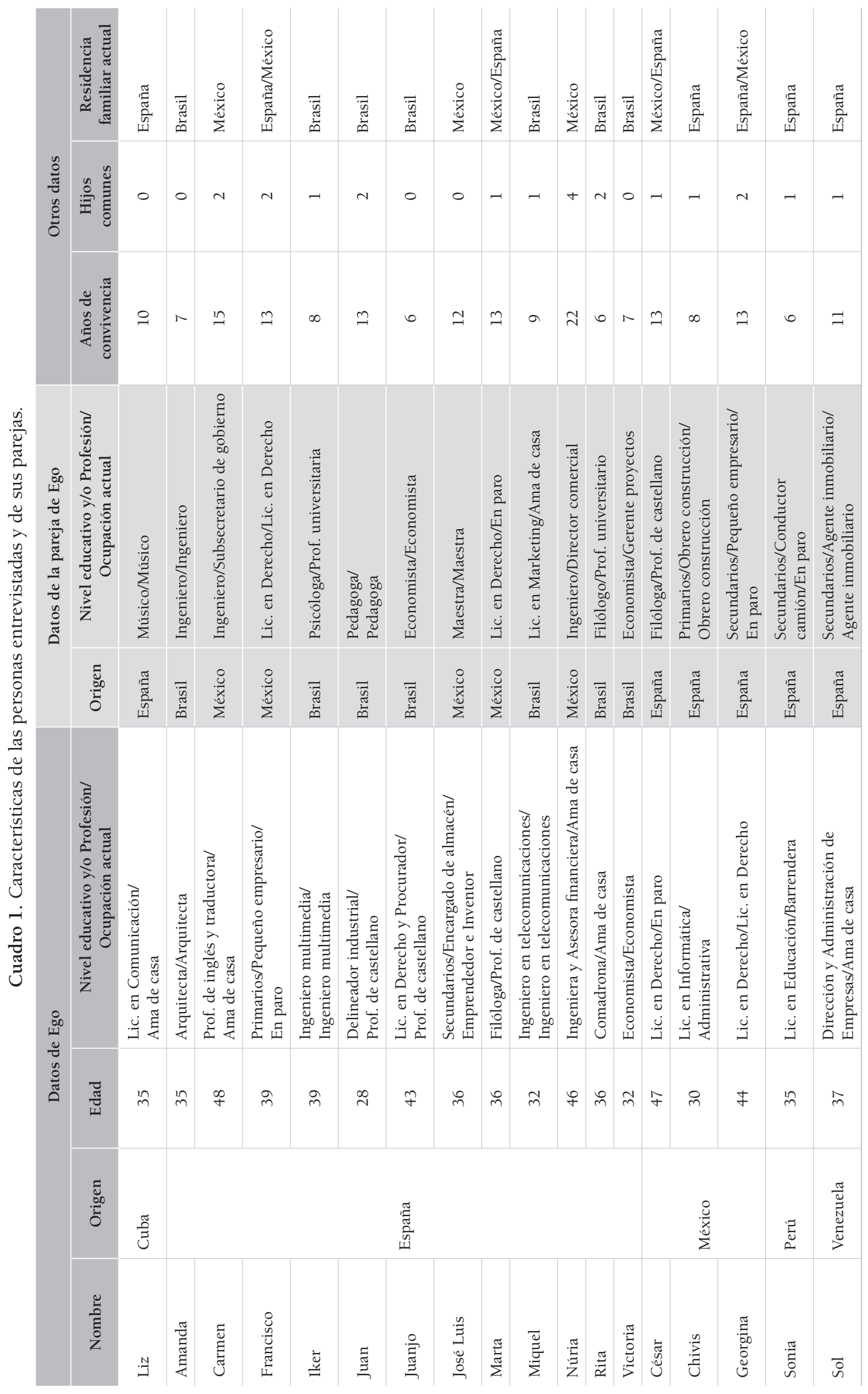
El tiempo de convivencia de las parejas entrevistadas oscila entre 6 y 22 años. De ellas, cinco no tenían hijos, siete tenían uno, cinco tenían dos y una tenía cuatro. Todas las mujeres de la muestra tienen formación universitaria. Las mujeres españolas están casadas en su totalidad con hombres con titulación superior, mientras que de los 11 hombres españoles casados con mujeres latinoamericanas cinco tienen estudios universitarios, cuatro tienen estudios secundarios y dos primarios. Hay, por lo tanto, seis parejas en las que el hombre español no cuenta con estudios superiores. Debemos ser cautos, por lo tanto, en la comparación de los procesos de incorporación a las sociedades de acogida de este grupo con los de las demás, debido a la diferencia en sus capitales culturales.

Como puede verse, se trata de una muestra bastante homogénea en términos del nivel de cualificación de los miembros de las parejas y en cuanto a su procedencia general: España y países de Latinoamérica, las dos variables fundamentales que abordamos en este trabajo. No sucede lo mismo en cuanto a la franja de edad, a los años de convivencia de la pareja en el momento de la entrevista, y al hecho de tener o no tener hijos y el número de ellos. Esta heterogeneidad resulta interesante porque permite observar con mayor nitidez las regularidades resultantes de las dos variables independientes consideradas y las diferencias encontradas y posiblemente articuladas con estas variables dependientes.

El análisis también se realizó de forma cualitativa mediante el recurso de la teoría fundamentada y la inducción analítica. Primero codificamos y organizamos las entrevistas transcritas de acuerdo con el lugar de origen de cada uno de los miembros de la pareja. Posteriormente creamos categorías en torno a las transformaciones en las trayectorias laborales de las personas entrevistadas y sus parejas, y la gestión y distribución de las responsabilidades domésticas. Habiendo comparado sus discursos y percepciones, identificamos los temas más significativos para una mejor comprensión y análisis a la luz de las aportaciones teóricas al respecto.

\section{RESULTADOS Y DISCUSIÓN}

Nos ocuparemos de analizar la incidencia de la migración en las trayectorias de personas a España desde distintos países latinoamericanos y también desde España a distintos países latinoamericanos. Por último, consideraremos el caso de dos mujeres que protagonizaron una doble migración, incluyendo una migración de retorno, entre Latinoamérica y España en un caso y viceversa en el otro.

\subsection{Contextos laborales}

\section{Parejas binacionales con destino España}

Entre las experiencias recogidas comprobamos que la situación laboral previa a la migración era satisfactoria. En el momento de tomar la decisión de dejar su país de origen para contraer matrimonio con un/a español/a, Sonia, Chivis, César y Liz tenían trabajo estable de acuerdo con su cualificación profesional. Sonia, a pesar de esto, y de que su pareja tuviera nivel de estudios menor, no se planteó la emigración de su futuro esposo. Sí que lo hicieron Liz y Chivis, que acabaron sucumbiendo no obstante a la convicción de las dificultades laborales que tendrían que padecer sus parejas masculinas en Cuba y México respectivamente. Ninguna de las dos dice haber ponderado los posibles inconvenientes que ellas mismas encontrarían para hacer lo propio en España.

\footnotetext{
Nunca tuve un conflicto en que yo fuera preparada y él no hubiera cumplido con sus estudios. Lo hablé con mi papá, inclusive, en un momento y se lo dije: "tenemos una cierta desventaja por lo que no es conveniente que se venga a México, por esta falta de preparación". [...] Inclusive él me decía: "Yo voy". Pero las oportunidades no las tenía, el horario... no parar en casa a mediodía, trabajar los sábados... entonces no daría para pagar el piso en México, el crédito de aquí (Chivis, México, 30) ${ }^{4}$.
}

Sonia y Sol, aunque con la decisión de migrar ya tomada, pactaron una prórroga con sus parejas para pasar un poco más de tiempo en su país de origen con el fin de poder cerrar adecuadamente cuestiones como la preparación emocional de sus familias, la finalización de sus estudios o de sus compromisos laborales.

\section{Parejas binacionales con origen España}

Un elemento que resultó determinante en los itinerarios laborales de buena parte de las parejas estudiadas fue la crisis económica global iniciada a finales de la década pasada. Esta generó, por un lado, el retorno de población inmigrada a sus países y, por el otro, la emigración de población española.

4 Después del nombre, señalamos país de origen y edad. 
A excepción de Núria, José Luis, Miquel y, en menor medida, Rita, las personas migrantes españolas de la muestra no descartaron la posibilidad ni incluso el deseo de regresar algún día a España, ya sea para toda la familia o para sus hijos/as. Así, Carmen -que emigró antes de la crisis- y Victoria -que lo hizo durante la crisis- han ido solicitando y renovando la excedencia laboral de sus trabajos en España, algo que no es frecuente encontrar entre los extranjeros que migraron a España en circunstancias similares.

Por ejemplo, Victoria, economista española de 32 años que conoció a su marido brasileño de 36 años en la empresa multinacional de Barcelona donde ambos trabajaban, cuenta de esta manera cómo se decidió:

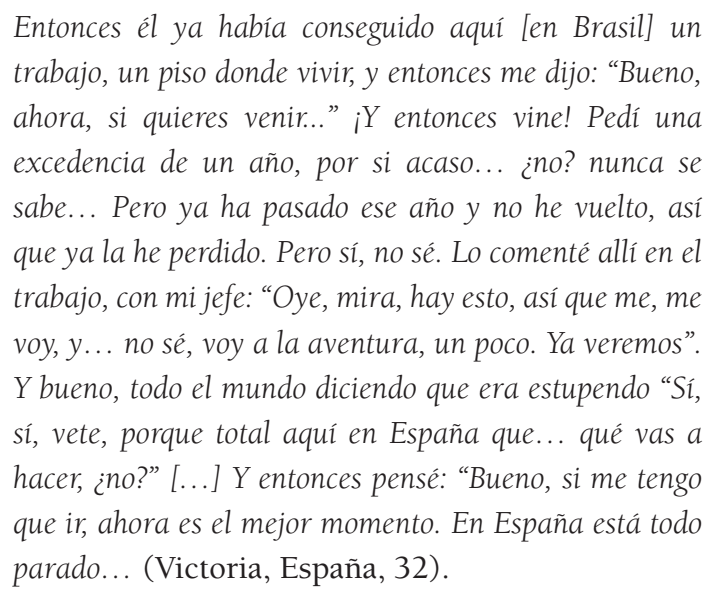

Carmen y Amanda, ambas españolas, demoraron un poco la decisión e incluso probaron, en un primer momento, a llevar la vida de pareja en España, pero finalmente acabaron cediendo. Todas las mujeres españolas de la muestra tomaron su decisión bajo el argumento de velar por las mejores perspectivas laborales de sus parejas, al igual que hicieron las mujeres extranjeras emparejadas con hombres españoles que hemos presentado antes. Tanto Carmen y Amanda, como Rita y Núria, priorizaron las oportunidades laborales -reales o potenciales- de sus parejas masculinas en sus respectivos países a sus propias oportunidades o realidades laborales en España. La estabilidad laboral de la pareja masculina detenta una posición preeminente e incuestionable en el razonamiento de nuestras protagonistas, tal como aparece expresado en el caso de Rita:

Es una persona estable, ¿sabes? Por un lado, es una persona estable, él tenía su trabajo, tenía sus cosas allá, o sea, que yo era un poco, hacía un poco de loca de dejar las cosas, lo mío, pero bueno, él tenía una estabilidad, ¿sabes? Yo dejaba, pero bueno, no es que me fuera... Yo pensaba así: "No es que me voy aquí, al vacio", ¿sabes? Él tiene una estructura y tal, ¿no? (Rita, España, 36).

Pero lo mismo sucede en los casos de hombres españoles como Iker, Juan y Juanjo: el primero emigró antes de la crisis sin mucho entusiasmo a Brasil, donde a su pareja le esperaba un puesto en la universidad, a pesar de que él podría haber seguido trabajando en la empresa en la que estaba.

Juan, delineador industrial, tenía un trabajo estable en su sector desde hacía 10 años, pero su pareja no encontraba posibilidades ciertas de ejercer su profesión, y decidieron irse a Brasil, de donde ella es originaria y donde sus contactos familiares le permitieron insertarse inmediatamente.

Juanjo, por su parte, dejó su propio gabinete de procuraduría para irse a Brasil, país de origen de su pareja. En España, a pesar de que el país ya estaba en plena crisis, tenía una vida muy cómoda (buen trabajo, buen sueldo, tiempo de ocio, etc.).

Entonces lo que yo me planteé fue: "Bueno, o sea, yo tengo un trabajo cómodo", tenía un trabajo cómodo, que me daba para vivir bien, vamos ibien! Bueno, yo no tengo cargas familiares, no tengo... En mi ciudad no hay grandes procedimientos judiciales, pero bueno ise gana un dinero! Yo vivía desahogado, todo a 5 minutos, con la moto voy a un sitio, voy a otro, voy a hacer deportes, ique me encanta! Lo tenía todo... (Juanjo, España, 43).

Pero decidió migrar porque quería facilitarle nuevas oportunidades laborales a su pareja, quien es economista y en España trabajaba de camarera. Ella logró insertarse laboralmente al poco tiempo de llegar a Brasil. En cambio, tanto él como Juan debieron reciclarse completamente (hacia la enseñanza del castellano), para lo que ambos tuvieron que hacer formaciones específicas.

En ocasiones, como en el caso de Victoria o de Francisco, la crisis económica aparece como argumento para justificar la decisión de emigrar desde su país, España, al de origen de la pareja.

Parejas binacionales entre España y América Latina

Dos de las parejas de la muestra realizaron estancias en los dos países de origen de cada uno de sus miembros, 
por lo que su consideración resulta de especial interés. La abogada Georgina, siguiendo el modelo ya relatado en el apartado anterior, dejó un trabajo estable y cualificado, familia, amigos y un piso en propiedad en Ciudad de México para casarse con un español con pocos estudios y un trabajo no cualificado y precario, con incursiones temporales en el paro. Para Georgina las consideraciones laborales propias no tuvieron mucho peso a la hora de decidir la emigración a España para emparejarse con Francisco, su marido. En cambio, cuando al cabo de nueve años regresó a México la razón no fue otra que las condiciones laborales de su marido, que fue quien tomó la decisión debido a la agudización de su precariedad laboral como consecuencia de la crisis económica. De hecho, ella se negaba a volver a México, pero la voluntad de su marido fue la que prevaleció. En la primera decisión de emigrar a España la interiorización del modelo familista, que sitúa a la mujer como responsable de la casa y los hijos y al marido como proveedor, hicieron gran parte del trabajo de legitimación de su apuesta. Y en la decisión del retorno a México, también. Como decía ella misma, hablando sobre su primera decisión de emigrar a España:

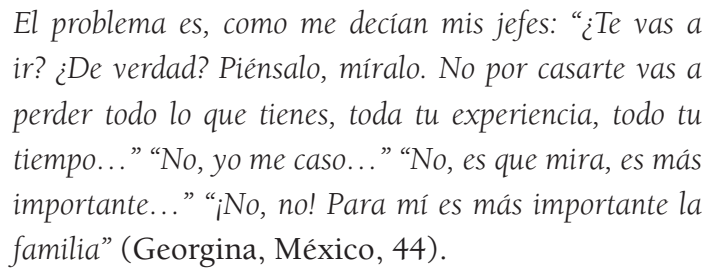

El itinerario de Marta pivota entre la misma dirección que el de Georgina, pero en un sentido inverso. La cuestión laboral, tanto de su marido -un alto directivo de la administración pública mexicana que ostentaba una buena posición laboral- como la suya, en el punto de empezar a desarrollar su itinerario profesional, fueron argumentos sólidos para decidir migrar a Ciudad de México: "Yo me fui con una maleta... Entonces, como que... pensé: 'Bueno, ¿qué vamos a hacer?' Él tenía un buen trabajo allá, yo no. 'Es más fácil que yo me quede en México..."'. Su aterrizaje en México no fue malo en términos laborales: trabajó como editora y dando clases de catalán. Pero a los tres años de su estancia, sintió que: "...la ciudad se me viene encima... Yo aquí no... no me veo viviendo toda la vida. César quería tener hijos, yo ahí no me veía...". La irrupción de un futuro escenario familiar con la llegada de los hijos en este caso hizo decidir a ambos, a instancias de ella, el regreso a España. En el momento de la entrevista ella trabajaba en dos lugares distintos, mientras que su marido, que no pudo convalidar sus estudios, iba encadenando períodos de paro con períodos de trabajo no cualificado.

La motivación laboral en este caso no está en la base de la toma de decisiones. Marta no tenía trabajo estable en España cuando emigró, pero sí que lo tenía en Ciudad de México cuando decidió regresar sin ningún trabajo en el horizonte en España. El retorno a España, no obstante, sí que tendrá graves consecuencias laborales para su esposo que, como en el caso anterior de Georgina, "lo deja todo":
Yo he roto tres grandes paradigmas de la vida y del mundo mundial, ¿eh? He roto el primer paradigma de: nadie deja su trabajo, nadie deja su ciudad, nadie deja sus amigos. [...] Ese es el primero. El segundo: nadie deja nada por una mujer. Es normalmente la mujer que sigue al hombre. Y yo he seguido a Marta. [...] Y... el tercer paradigma: como México no hay dos. [...] Y yo pensaba esto: "México, México no hay dos. Es que como en México, no se vive en ningún lado". Pues tienes razón. Y no al mismo tiempo, ¿no? A veces se vive mejor, a veces se vive peor... (César, México, 47).

Vista la incidencia de la dimensión laboral en las decisiones migratorias de nuestras parejas, veamos ahora cómo el hecho migratorio incidió en el balance entre lo laboral y lo doméstico en sus miembros.

\subsection{Migrar por amor y redefinir lo laboral y lo doméstico}

Cuando hablamos de emigración por amor, como hemos venido haciendo a lo largo de este artículo, estamos subrayando que el motivo principal -ya sabemos desde hace tiempo que nunca hay un solo motivo en un proyecto y/o proceso migratorio- de la movilidad protagonizada por las personas así etiquetadas es el amor, el deseo de iniciar una relación sentimental con otra persona de -y residente en- otro país. Cuando la razón migratoria principal es la laboral ya resulta a menudo difícil la inserción laboral en destino, aunque en mucha menor medida si se trata de una emigración laboral cualificada, que a veces ni se concibe como migración, por lo que los "migrantes" cualificados son llamados en ciertos ámbitos "expatriados". En el caso que nos ocupa, el escenario resulta aún más complejo. Hablamos de personas cualificadas, generalmente con 
una buena posición laboral en sus países de origen, que deciden abandonar su país, y con él su trabajo, por razones amoroso-sentimentales. A pesar de que la consideración de la dimensión laboral, como hemos visto, no está exenta de los elementos tomados en cuenta por ambos miembros de la futura pareja, lo cierto es que en la mayor parte de casos se mantiene en un segundo plano. Por tanto, la incorporación laboral en destino resulta más complicada que en el caso de que ella hubiera sido el motivo principal de la movilidad. Desde hace un tiempo, además, estas dificultades aplican tanto a desplazamientos realizados del Sur al Norte global como a la inversa, dado que muchos países del Sur global han ido endureciendo las condiciones legales de acceso al trabajo de las personas inmigrantes, tal como venían haciendo los países del Norte global.

Comparadas con los emigrantes de carácter económico, las personas de la muestra han gozado de ciertas ventajas en la incorporación a sus lugares de destino de la mano de un matrimonio con un miembro nacional (Bodoque y Soronellas, 2010). Primero, porque dicho matrimonio va asociado a ciertas ventajas legales que puede simplificar el acceso al mercado laboral. Y segundo, porque les facilita compartir la red social y profesional de su pareja, lo cual puede resultar un factor relevante para la inserción laboral.

Esta encrucijada de elementos favorables y desfavorables a la incorporación laboral se conjuga de manera diversa en la experiencia de las personas que constituyen nuestra muestra, y tiene a su vez consecuencias igualmente diversas en la (re)organización de la gestión del ámbito doméstico de la pareja.

La totalidad de las personas provenientes de América Latina que hemos entrevistado llegaron a España ya casadas o justo para casarse a su llegada. En el caso de las personas españolas que emigraron de España, por el contrario, encontramos una diversidad de situaciones. Algunas de ellas no tenían la intención inicial de casarse, aunque la mayoría acabó haciéndolo porque, entre otras cosas, ello facilitaba la residencia y las posibilidades de acceso al mercado de trabajo, como han señalado para distintos países Piper y Roces (2003).

Así, por ejemplo, Marta se casó cuando no pudo renovar más su visado de turista en México, "y al poco tiempo encontré trabajo". Victoria se casó en Brasil en mayo de 2012, con lo cual obtuvo el permiso de trabajo, y seis meses después comenzó a trabajar. Como ella misma nos contó, "casarse lo facilita todo", como ya le había confesado en una entrevista de trabajo un directivo de una multinacional catalana: “...y él me dijo, así, claramente: 'Cásate. Cásate, olvídate de todo lo demás, y va a ser mucho más fácil'". Evidentemente se trata de un factor más. En su caso reconoce que le ayudó también el tener una formación superior y el conocimiento de idiomas. Pero como ya indicábamos, las situaciones y sus correspondientes experiencias son variadas. Victoria, por su parte, no ha conseguido un trabajo que concuerde con su formación: es economista y como tal trabajaba en Barcelona, mientras que en Rio de Janeiro trabaja en un despacho de abogados y se encarga de la comunicación con los clientes extranjeros.

En los casos más afortunados nos encontramos con algunas de nuestras protagonistas que sí encontraron un trabajo acorde a su titulación, pero generalmente con unas condiciones laborales no comparables con las que tenían en sus trabajos en España. Es el caso de Amanda, una arquitecta que emigró a Brasil para vivir con su pareja en Belo Horizonte y posteriormente decidió buscar trabajo a $500 \mathrm{~km}$, en Rio de Janeiro, porque no le gustaba Belo Horizonte y sintió que en Rio tendría más oportunidades. La contrató un joven y pequeño despacho de arquitectura:

\footnotetext{
...y la verdad que estoy contenta. La única pega es que cobramos mal, muy poco, ya te dicen: "cartera de trabalho". Yo cobro 3.000 reales, unos mil y poco euros. Estoy compartiendo piso con una pareja de brasileños [su cónyuge sigue viviendo en Belo Horizonte], y pago 1.100 reales por la habitación... (Amanda, España, 35).
}

Es distinta la situación de Miquel, quien se fue a Brasil (país de origen de su pareja) con un contrato de trabajo que le entusiasmaba y satisfacía. Y lo es también -pero en sentido opuesto- la de Francisco, quien después de haber sido propietario de una pequeña empresa en España, en México pasa largos períodos en paro alternados con contratos de poca duración.

Además, varios de estos hombres españoles llegaron a sus nuevos países de residencia con proyectos de emprendimiento: se trata del mismo Francisco, Juan, Juanjo y José Luis. Sólo Juanjo consiguió concretar alguno de sus proyectos; los otros se frustraron debido a problemas burocráticos, falta de red social, falta de financiación o motivación, etc. Por otro lado, dos de 
ellos (Juan y Juanjo) se terminaron "reconvirtiendo", como ya mencionamos, en profesores de castellano.

En el caso de las mujeres latinoamericanas cualificadas de la muestra que emigraron por amor a España, este proceso de descualificación laboral se radicalizó, y las situaciones de paro eran habituales. La cubana Liz, después de siete años viviendo en España, no tenía aún un trabajo remunerado. Al igual que la venezolana Sol, que se dedicaba a las tareas domésticas y de cuidado.

Irónicamente, mientras que algunas de estas mujeres originarias del sur global se casan internacionalmente para huir de los roles de género tradicionales (Roca Girona, 2011), sufren una descualificación que, en muchos casos, las confina al nicho doméstico (Piper y Roces, 2003).

Sonia, una mujer peruana con titulación universitaria, después de más de una hora de entrevista, nos confesó:

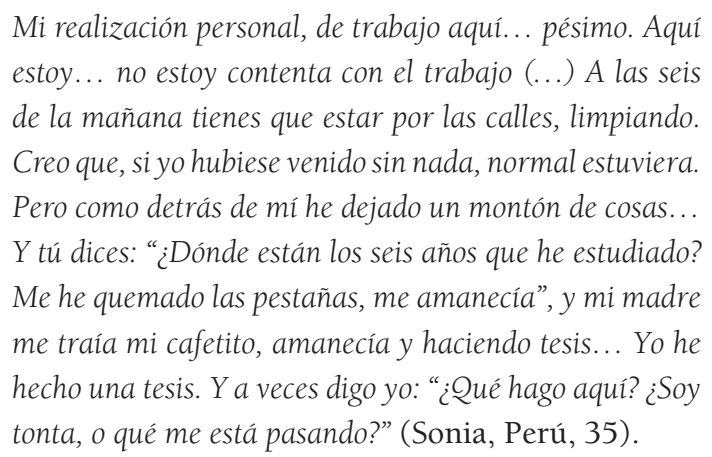

Por otro lado, Georgina, la abogada mexicana, en el período de nueve años que vivió en España nos contó que realizó todo tipo de trabajos esporádicos no cualificados, desde limpiadora en el sector turístico a reponedora en centros comerciales.

\section{Cuando llegan los hijos...}

Existe un acuerdo generalizado en señalar que la articulación entre la esfera laboral y la reproductiva en el marco de una pareja deviene un asunto especialmente problemático cuando aparece en el escenario la crianza de los hijos, con la correspondiente incidencia en las relaciones de género y las trayectorias laborales de los miembros de la pareja.

Cuando Carmen llegó a México desde España, hace casi diez años, con su hija recién nacida, había decidido que se dedicaría a su crianza durante los tres años siguientes. Finalizado ese período, tenía la intención de reincorporarse a su trabajo como profesora, del que había solicitado excedencia en España. Transcurridos estos tres años, se encontró con que se había desatado la crisis económica y que su marido había alcanzado en México una muy buena posición laboral. Y se había quedado embarazada, además, de su segunda hija.

La mayor tiene once años, está en quinto de primaria. Pero yo, como veo las cosas allí, ahora con la crisis está mal, ahora está peor. Él no va a encontrar un trabajo mejor. Y ya me acostumbré. Aquí como que tengo miedo, digo que llevo mucho tiempo sin trabajar y claro, ¿y dónde voy? Dar clase, tal y como están aquí las escuelas no me apetece, a parte que cobran fatal (Carmen, España, 48).

Entre los hombres, podemos citar a Francisco, quien, debido a su situación de desempleo y a las largas horas de trabajo de su esposa, tuvo que involucrarse -aunque poco y a disgusto- en el cuidado de sus hijos. Por el contrario, Iker propuso a su pareja brasileña quedarse en casa a cuidar del hijo común, aunque ella no aceptó:

\footnotetext{
Y ahí, yo ya lo dije que no me importaría quedarme en casa y cuidarle yo, pero la cosa aquí todavía no está... no hay una mentalidad tan abierta aquí como para que... o bueno, que Katia entendiera eso... o bueno, o yo qué sé, no sé. Alguna cosa ahí pasaba. Porque por dinero no fue. Ella tiene muy buen trabajo y podríamos haber estado bien con un sueldo tranquilamente viviendo bien. Pero... como que yo tenía que seguir trabajando... (Iker, España, 39).
}

Ambos ejemplos resultan interesantes, ya que muestran actitudes contrapuestas, por parte de dos hombres españoles con la misma edad (39 años), pero con nivel de estudios y cualificación laboral muy distintos, sobre las relaciones de género. En el caso de Francisco constatamos una actitud reacia a asumir las responsabilidades en la esfera doméstica a pesar de encontrarse desempleado y con su esposa Georgina (que nos contó que en México resulta habitual que los hombres realicen tareas domésticas) laboralmente activa. En el caso del ingeniero Iker, su actitud de disponibilidad en dejar el trabajo remunerado y asumir las responsabilidades domésticas choca con las expectativas de género de su pareja Katia, incómoda ante un reparto de tareas que aparentemente no refleja el modelo mayoritario en Brasil. Ambas mujeres son 
altamente cualificadas: Georgina es una abogada mexicana de 44 años, y Katia es una psicóloga y profesora universitaria brasileña de 35 años.

César, por su parte, vivía una situación parecida a la de Francisco -aunque él decía disfrutarla- ya que, debido a las largas jornadas laborales de su esposa y su propio desempleo, él se hizo cargo del hijo de ambos.

En otros casos la crianza de los hijos se compagina con el trabajo extra-doméstico remunerado, a menudo con escasa participación del miembro masculino en la esfera reproductiva. Rita, la comadrona española de 36 años que desarrollaba su trabajo en España organizándolo en 9 meses de carga intensiva y 3 meses que aprovechaba para viajar por el mundo, tuvo claro desde el primer momento que se dedicaría casi en exclusiva a la crianza de sus dos hijos. Y nos subrayó enfáticamente que se trataba de una elección libre y voluntaria, de acuerdo con lo descrito para una situación similar por Purkayastha (2005):

Los niños estaban muy pequeños, y yo he querido, he elegido ¿sabes?, he elegido estar con ellos, entonces a mí no me incomoda nada, porque precisamente he podido estar con ellos sin tener la presión de que tengo un horario para entrar a trabajar y otro para salir. Eso lo tiene él. Yo me he acogido a ese rol tradicional. Él me dice: "iTú puedes trabajar! ¡Trabaja!”, no sé qué. ¡Pero yo no quiero! Si yo trabajo, ¿quién se queda a cuidar de ellos? Están pequeñitos, y yo no quiero dejarlos en manos... ¿Cómo voy a dejar esto, que para mí es tan importante, en manos de alguien que no sé quién es? (Rita, España, 36).

El trabajo extra-doméstico aparecía en el horizonte vital de Rita tan sólo como una distracción, y con un peso poco sustancial. De hecho, pasa períodos en España durante los cuales ejerce su profesión mientras ella y sus hijos conviven con su familia de orientación.

Resultan remarcables las situaciones semejantes del ingeniero Iker y de la comadrona Rita, ambos de nacionalidad española, emparejados con personas de nacionalidad brasileña, profesores de universidad, y viviendo en Brasil, y las resoluciones antagónicas en ambos casos. Tanto Iker como Rita quieren asumir las responsabilidades domésticas a tiempo completo. Iker tiene que lidiar con la oposición de su pareja brasileña, profesora de Psicología, a la que incomodaría si él no tuviera un trabajo remunerado, mientras que Rita, que sí consigue dedicarse a la esfera doméstica en exclusiva cuando está viviendo en Brasil, debe convivir con el hecho de que su pareja, profesor de Filología, le anime constantemente a realizar algún trabajo extradoméstico.

Finalizamos nuestro periplo con las experiencias particulares de migración y re-migración de Georgina y Marta. Georgina, tras encadenar trabajos no cualificados y precarios durante su estancia en España, se vio obligada a regresar a México debido a la decisión de su marido español, que pensó que sería una buena estrategia para mejorar la situación laboral de paro que llevaba arrastrando desde hacía tiempo. A su no deseo de retornar a México se unía la perspectiva de unas expectativas laborales, a su parecer, nada halagüeñas. Poco después de regresar, no obstante, Georgina encontró un trabajo acorde a su titulación en la administración pública. Sin embargo, cree que la probabilidad de que su marido, un trabajador no cualificado, consiga un trabajo estable es muy baja. Tanto como la de que se implique en las tareas domésticas y de cuidado en las que ya en España nunca participó. Razón por la cual ella ya tuvo claro qué tipo de trabajo debía buscar a su llegada a la capital mexicana:

\begin{abstract}
Lo que yo quiero es un trabajo donde yo pueda compartir con mis hijos momentos... El hecho de a la tarde poder estar con ellos, hacer la tarea... Y no irme en la mañana y regresar a la noche y que no me vean. Y, además, al venir aquí -a México-, como que están cambiando un poco los papeles, y sí, iyo siento que... Dicen que los mexicanos son machistas... comparados con los españoles! Como que a Francisco le ha costado mucho el tener que admitir que esta vez a lo mejor soy yo la que puedo tirar del carro, ¿no? Y le cuesta mucho, ¿eh?, parece que se le está cayendo el mundo encima. Le digo. "Pues no tiene nada de malo, ¿no? Nosotros somos una pareja, una familia, y para eso estamos." Le cuesta... (Georgina, México, 44).
\end{abstract}

La situación descrita hasta aquí cambia de forma radical en las vivencias de Marta y César. En este caso se invierten la nacionalidad y el género de los miembros de la pareja -siendo ella la española y él el mexicano-, así como el itinerario migratorio -de España a México y de allí a España nuevamente. Pero no sólo eso. El proceso adaptativo de la parte masculina de la pareja es absolutamente antagónico: 
Bueno, eso [que César no haya podido convalidar los estudios, que haya tenido que buscar trabajo en otro sector, y que esté en el paro] ya lo sabíamos de alguna manera. ¡César tenía un buen trabajo! Su carrera aquí no se la convalidaban. César llegó un día que me dijo: "Si tú aquí [en México] no vas a estar bien, yo no voy a estar bien.' Dice: "Voy allá [a España], y aunque tenga que descargar camiones, de algo trabajaré." Trabajó de consultor y comercial, y después de técnico de compras. [...] Y luego llegó el niño. Lo cuida él, porque al estar desempleado, es papá completo, día completo (Marta, España. 36).

De manera general constatamos que la mayoría de las personas entrevistadas se debaten entre planteamientos antagónicos. Por un lado, tenemos que la mayoría, tanto mujeres como hombres, tras un proceso migratorio marcado en ocasiones por la crisis económica, intentan continuar su carrera laboral con la misma orientación que en el país de origen. Pero por otro lado nos encontramos con que las mujeres con hijos asumen una mayor o exclusiva dedicación a la crianza, mientras en los hombres se produce una reconversión profesional, así como -en algunos casosuna mayor dedicación a las tareas de cuidado.

Por lo que respecta a la intención de continuar con la carrera profesional, se puede afirmar que su elevada preparación y su experiencia profesional previa son los principales argumentos. Por lo que respecta a las reorientaciones, su migración de naturaleza amorosa, no laboral, su condición de inmigrantes y su edad (que sitúa a las mujeres en fases críticas de la reproducción biológica) serían los principales elementos para tener en cuenta.

\section{CONCLUSIONES}

Las personas que conforman la muestra seleccionada son mayoritariamente cualificadas, con formación universitaria, que tras conocer a una persona de otra nacionalidad decidieron iniciar una relación que supuso su emigración al país de su pareja. Estas parejas tienen varios aspectos en común: son heterosexuales; están formadas por un miembro español -masculino o femenino- y otro de alguna nacionalidad latinoamericana (grupo mayoritario de personas extranjeras emparejadas con personas españolas); y su proceso migratorio tiene como origen o destino España. Tenemos pues dos grandes grupos de migrantes por amor: el compuesto por personas que llevan a cabo una emigración desde América Latina a España, que constituye un colectivo ampliamente estudiado, y el compuesto por personas con un itinerario migratorio de sentido inverso, un colectivo escasamente estudiado por la literatura especializada.

Nuestros hallazgos deben ser contextualizados con la naturaleza de la muestra sobre la que hemos trabajado, donde encontramos dos variables compartidas -la condición de personas altamente cualificadas laboralmente hablando y su matrimonio con una persona de otra nacionalidad, con la consiguiente migración de uno de los miembros de la pareja- y otras diferenciales -como por ejemplo los años de relación de pareja, la existencia o no de hijos, la edad, la nacionalidad, etc.Si bien esto no nos permite generalizar los resultados obtenidos, sí que nos permite mostrar, por el contrario, una variedad de semejanzas y diferencias que enriquecen el conocimiento sobre las cuestiones que hemos abordado.

Los trabajos publicados sobre diferentes aspectos parciales de los sujetos de estudio resaltan el hecho que, en estas situaciones, la mayoría de las mujeres relegan a un segundo plano sus proyectos laborales y priman los de sus compañeros en su toma de decisiones. Esto es algo que hemos podido corroborar en el relato de la mayoría de nuestras informantes. Carmen, Núria, Amanda y Rita emigraron de España a México, las dos primeras, y a Brasil las dos últimas, dejando atrás una buena posición profesional. Y en sentido inverso, migrando hacia España, también lo hicieron Chivis y Georgina desde México, Sonia desde Perú y Liz desde Cuba. La influencia del artefacto construido en torno al binomio producción-reproducción y la correspondiente segregación de género asociada a éste parece que sigue estando vigente. El amor, para muchas de estas mujeres, parece pasar por delante de su trabajo remunerado y sus aspiraciones profesionales, mientras que para la mayoría de los hombres considerados ocurre lo contrario. Y es cierto que muchos de ellos, con mayor o menor sutileza, pusieron a sus parejas en la disyuntiva de acabar con la relación sentimental si no realizaban un proceso migratorio. Esto, desde luego, sitúa en una posición ventajosa a los hombres, que juegan en campo propio, en el terreno de las dinámicas y las verdades dadas por descontado, como dirían Berger y Luckmann (2003), mientras que las 
mujeres deambulan por un terreno frágil y pantanoso ya que no están familiarizadas con esta cultura. Una de las consecuencias de esto es que el capital social y cultural de las mujeres mengua, en tanto que el de sus parejas no varía o lo hace en menor medida, incluso de forma ascendente a veces. Estas trayectorias ponen en evidencia a mujeres que "lo dejaron todo por amor", incluida su posición y aspiraciones laborales que, con la emigración, se vieron seriamente dañadas, frustradas o simplemente abandonadas.

No obstante, las experiencias de nuestras y nuestros informantes nos presentan diversos ejemplos de transgresión de esta suerte de norma. Y nuestro trabajo aporta la constatación novedosa de procesos escasamente estudiados de migración amorosa masculina. Más concretamente, de una vertiente de ella no abordada aún por la literatura especializada, como es la migración por amor de hombres del Norte global al Sur global. Y así nos encontramos con procesos similares a los descritos arriba para la mayoría de las mujeres. Iker, Juan y Juanjo dejaron posiciones laborales altamente cualificadas en España y emigraron a Brasil, el país de sus respectivas parejas.

En algunos casos esa migración masculina por amor se produce en una fase posterior a la inicial, y más habitual, de la mujer al país de residencia del hombre. Es el caso de las parejas formadas por Francisco y Georgina, y por Marta y César. La situación de los primeros se explica fácilmente por la diferencia de nivel de formación entre ambos. La segunda pareja, sin embargo, es más atípica y los protagonistas lo explican por las dificultades de adaptación de ella a una gran ciudad como es México DF, combinado con su deseo de tener hijos y de formar una familia en un entorno más propicio para ellos. En estos dos casos, además, podríamos ensayar incluso algún tipo de consideración de los sistemas de género característicos de ambos países para interpretar, por un lado, el papel jugado por los cuatro actores y, por otro, los matices y deconstrucciones que estas experiencias aportan a la hora de dar por sentada la naturaleza, y su incidencia, de los sistemas de género del Sur y el Norte global. Con la mexicana Georgina adoptando un papel de clara subordinación respecto a su marido español, que implica su emigración a España primero y su retorno a México contra su voluntad después. Y con su marido español, que ni en España ni en México (a pesar de su habitual situación de desempleo) asume ninguna responsabilidad en las cuestiones de la crianza de los hijos y del trabajo doméstico. Y la española Marta, que accede en primera instancia a emigrar al país de su marido, pero que posteriormente plantea su voluntad de regresar a España. Algo que su marido mexicano asume y apoya, con las consiguientes consecuencias negativas -las mismas que hemos podido constatar para la mayoría de las mujeres de la muestra- para su balance laboral-reproductivo.

La experiencia de César entronca con la de los casos que ya hemos señalado en que la migración por amor de la pareja masculina al país de la femenina resulta ser la primera opción, ya que en la mayoría de estos casos de migración masculina por amor los hombres migrantes se ven afectados también por consecuencias similares a las que hemos señalado para las mujeres. Es decir, dificultad de incorporación al mercado laboral, disminución de ingresos, mengua de perspectiva de desarrollo profesional, menor cualificación de los trabajos encontrados, subempleo o el desempleo. Bien puede afirmarse que la familia agregada puede considerarse como un peso en este tipo de migración. El paradigma neoliberal de "capital humano" ignora el rol de la familia en la migración.

En el caso de los hombres se produce también un incremento de participación y responsabilización de las tareas reproductivas, básicamente las de tipo doméstico y las relativas al cuidado y crianza de los hijos, pero mucho menor que el comprobado entre las mujeres. Hemos podido constatar además que las mayores resistencias no provienen necesariamente de hombres procedentes del Sur global, sino que la procedencia del Norte global no es garantía de una mejor disposición y actitud masculina para asumir mayores responsabilidades domésticas. De igual modo, en ocasiones, la disponibilidad masculina a una mayor implicación en la esfera reproductiva se ve frenada en parte por el sistema de género predominante en la sociedad receptora, como ejemplifica el caso de Iker y Katia.

Recuperando la hipótesis que planteábamos al inicio, podemos concluir que la trayectoria laboral que realmente se verá significativamente afectada en términos de descualificación y/o reorientación será la del miembro de la pareja que lleva a cabo la emigración, con independencia de que se trate de la 
mujer -lo más habitual- o del hombre. Por lo que a la implicación en las responsabilidades domésticas se refiere, las mujeres siguen asumiéndolas en mayor grado, independientemente de sus situaciones laborales y/o las de sus parejas, y de que sean ellas las migrantes o no. Aunque esta asunción debe correlacionarse también con la variable de la clase social, no tanto en el sentido de la responsabilidad y gestión como de la realización, ya que en parejas binacionales altamente cualificadas de altos ingresos -lo cual no siempre va en consonancia- acostumbra a externalizarse con personal contratado.

Todo ello nos conduce a concluir en la necesidad de aplicar una mirada interseccional al análisis de este tipo de realidades, teniendo en cuenta el cruce de variables fundamentales como la edad, la presencia o no de hijos, la nacionalidad de nuestras y nuestros protagonistas y de sus parejas, el nivel educativo y la clase social, los años de convivencia, la condición o no de migrante, el país de residencia, así como el sentido y la duración de la migración.

Desde luego, la propensión popular a percibir los matrimonios binacionales como "interesados" y la de muchos gobiernos a conceptualizarlos como fraudulentos no dejan de ser burdas simplificaciones que ignoran su complejidad y las dificultades que en muchos aspectos entrañan.

\section{REFERENCIAS BIBLIOGRÁFICAS}

Aure, M. (2013). Highly skilled dependent migrants entering the labour market: Gender and place in skill transfer. Geoforum, 45, 275-284. https://doi.org/10.1016/j. geoforum.2012.11.015

Beck, U. y Beck-Gernsheim, E. (2012). Amor a distancia. Barcelona: Paidós.

Beiruti, N. (2008). La inmigración en la encrucijada de la adaptación. Norte de Saludmental. http://www.revistanorte. es/index.php/revista/article/download/561/555

Berger, P.L. y Luckmann, T. (2003). La construcción social de la realidad. Buenos Aires: Amorrortu.

Bodoque, Y. y Soronellas, M. (2010). Parejas en el espacio transnacional. Los proyectos de mujeres que emigran por motivos conyugales. Migraciones Internacionales, 5, 143174. Recuperado en 13 de enero de 2021, de http://www. scielo.org.mx/scielo.php?script=sci_arttext\&pid=S1665$89062010000100005 \& \operatorname{lng}=\mathrm{es} \& \ln \mathrm{ln}=\mathrm{es}$

Breger, R. y Hill, R. (Eds.) (1998). Cross-Cultural Marriage. Oxford: Berg.

Cam Thai, H. (2002). Clashing Dreams: Highly educated overseas brides and low-wage U.S. husbands. En: B.
Ehrenreich y A. R. Hochschild (Eds.) Global Woman. (pp. 230-253). London: Granta Books.

Cam Thai, H. (2005). Clashing Dreams in the Vietnamese diaspora: Highly educated overseas brides ans low-wage U.S. husbands. En: N. Constable (Ed.) Cross-Border Marriages. (pp: 145-165). Philadelphia: University of Pennsylvania Press.

Constable, N. (Ed.) (2003). Romance on a Global Stage. Berkeley: University of California Press.

Cooke, F.L. (2007). Husband's career first: Renegotiating career and family commitment among migrant Chinese academic couples in Britain. Work, Employment and Society, 21, 47-65. https://doi.org/10.1177/0950017007073615

Charsley, K. (Ed.) (2012). Transnational Marriage. New Perspectives from Europe and Beyond. New York: Routledge.

Dumont, J-C., Martin, J.P. y Spielvogel, G. (2007). Women on the Move: The Neglected gender dimension of the brain drain. https://www.iza.org/publications/dp/2920/womenon-the-move-the-neglected-gender-dimension-of-thebrain-drain

Hardill, I., y MacDonald, S. (1998). Choosing to recolocate: An Examination of the impact of expatriate work on dualcareer households. Women's Studies International Forum, 21, 21-29. https://doi.org/10.1016/S0277-5395(97)00085-X

Harris, J.R. y Todaro, M.P. (1970). Migration, unemployment and developmnent: A two-sector analysis. The American Economic Review, 60(1), 126-142. Recuperado en 13 de enero de 2021, de: https://www.jstor.org/stable/1807860

Ho, C. (2006). Migration as feminisation? Chinese women's experiences of work and family in Australia. Journal of Ethnic and Migration Studies, 32(3), 497-514. https://doi. org/10.1080/13691830600555053

Ho, C. y Alcorso, C. (2004). Migrants and employment. Challenging the success story. Journal of Sociology, 40(3), 237-259. https://doi.org/10.1177/1440783304045721

Illouz, E. (2012). Por qué duele el amor. Madrid: Katz.

Iredale, R. (2000). Migration Policies for the Highly Skilled in the Asia-Pacific Region. International Migration Review, 34(3), 882-906. https://doi. org/10.1177/019791830003400309

King, R. (2002). Towards a new map of European migration. International Journal of Population Geography, 8, 89-106. https://doi.org/10.1002/ijpg.246

Kofman, E. (2000). The invisibility of skilled female migrants and gender relations in studies of skilled migration in Europe. International Journal of Population Geography, 6(1), 45-59. https://doi.org/10.1002/(SICI)10991220(200001/02)6:1<45::AID-IJPG169>3.0.CO;2-B

Man, G. (2004). Gender, work and migration: Deskilling chinese immigrant women in Canada. Women's Studies International Forum, 27(2), 135-148. https://doi. org/10.1016/j.wsif.2004.06.004

Marcus, G.E. (1995). Ethnography in/of the World System: The Emergence of multi-sited ethnography. Source: Annual Review of Anthropology, 24, 95-117. https://doi. org/10.1146/annurev.an.24.100195.000523

Meares, C. (2010). A fine balance: Women, work and skilled migration. Women's Studies International Forum, 33(5), 473-481. https://doi.org/10.1016/j.wsif.2010.06.001 
Penny, J. y Khoo, S. (1996). Intermarriage. A Study of migration and integration. Canberra: Australian Government Publishing Service.

Piper, N. y Roces, M. (Eds.). (2003). Wife or worker?: Asian women and migration. Lanham, Maryland: Rowman \& Littlefield Publishers.

Purkayastha, B. (2005). Skilled migration and cumulative disadvantage: The case of highly qualified Asian Indian immigrant women in the US. Geoforum, 36(2 SPEC. ISS.), 181-196. https://doi.org/10.1016/j. geoforum.2003.11.006

Riaño, Y. y Baghdadi, N. (2007). 'Je pensais que je pourrais avoir une relation plus égalitaire avec un Européen.' Le rôle du genre et de l'imaginaire géographique dans la migration des femmes. Nouvelles Questions Féministes, 26(1), 38-53. https://doi.org/10.3917/nqf.261.0038

Robinson, K. (2007). Marriage migration, gender transformations, and family values in the 'Global Ecumene', Gender, Place \& Culture, 14(4), 483-497. https://doi.org/10.1080/09663690701439793

Roca Girona, J. (2007). Migrantes por amor. La búsqueda y formación de parejas transnacionales. AIBR. Revista de Antropología Iberoamericana, 2(3), 430-458. https://www. redalyc.org/articulo.oa?id=623/62320303

Roca Girona, J. (2011). [Re]buscando el amor: Motivos y razones de las uniones mixtas de hombres españoles con mujeres extranjeras, Revista de Dialectología y Tradiciones Populares, 64(2), 487-514. https://doi.org/10.3989/ rdtp. 2011.18

Roca Girona, J., Soronellas, M y Bodoque, Y. (2012). Migraciones por amor: diversidad y complejidad de las migraciones de mujeres. Papers (revista de Sociología), 97(3), 685-707. https://www.raco.cat/index.php/Papers/ article/view/255856

Varrel, A. (2011). Gender and intergenerational issues in the circulation of highly skilled migrants: The case of Indian IT professionals. En: A. Kraler, E. Kofman, M. Kohli y C. Schmoll (Eds.), Gender, generations and the family in international migration. (pp. 335-354). Amsterdam: Amsterdam University Press. http://library.oapen.org/ handle/20.500.12657/34532

Williams, L. (2010). Global Marriage. Cross-border marriage migration in a global context. London: Palgrave Macmillan.

Willis, K. y Yeoh, B. (2003). Gender, marriage, and skilled migration: The Case of Singaporeans in China. En: N. Piper y M. Roces (Eds.), Wife or worker?: Asian women and migration. (pp. 101-119). Lanham, Maryland: Rowman \& Littlefield Publishers.
Yeoh, B.S. y Willis, K. (2005). Singaporeans in China: Transnational women elites and the negotiation of gendered identities. Geoforum, 36(2), 211-222. https:// doi.org/10.1016/j.geoforum.2003.07.004

\section{NOTAS BIOGRÁFICAS}

\section{JORDI ROCA GIRONA}

Doctor en Antropología y Catedrático de Antropología Social del Departamento de Antropología, Filosofía y Trabajo Social de la Universitat Rovira i Virgili de Tarragona. Sus líneas de investigación se centran en los estudios de género, la sexualidad y el amor, así como en diversas cuestiones relacionadas con la memoria biográfica, la etnografía y los estudios organizacionales.

\section{CLAUDIA MARÍA ANLEU-HERNÁNDEZ}

Doctora en migraciones y mediación social por la Universitat Rovira i Virgili. Máster en Psicología Social y Violencia Política (Universidad San Carlos de Guatemala) y Máster en Migraciones y Mediación Social (URV). Es investigadora post doctoral en el Departamento de Antropología, Filosofía y Trabajo Social de la URV. Sus líneas de investigación se centran en resiliencia, migraciones, trabajo e intervención social, parejas mixtas y juventud.

\section{VERÓNICA ANZIL}

Doctora en Antropología por la Universitat Rovira i Virgili. Licenciada en Antropología (Universidad Nacional de La Plata, Argentina). DEA en Sociología, orientación Sociologie de la Culture et du Changement Social, en la Universidad Paris VII-Jussieu (Paris, Francia). Sus principales líneas de investigación y publicación son: familias transnacionales, adopción transnacional, parejas binacionales, identidades y estereotipos. 